\title{
Blocking sets of tangent lines to a hyperbolic quadric in $\mathrm{PG}(3,3)$
}

\author{
Bart De Bruyn Binod Kumar Sahoo Bikramaditya Sahu
}

September 4, 2017

\begin{abstract}
Let $Q^{+}(3, q)$ be a hyperbolic quadric in $\operatorname{PG}(3, q)$ and $\mathcal{T}$ be the set of all lines of $\operatorname{PG}(3, q)$ which are tangent to $Q^{+}(3, q)$. If $k$ is the minimum size of a $\mathcal{T}$-blocking set in $\operatorname{PG}(3, q)$, then we prove that $q^{2}+1 \leq k \leq q^{2}+q$. When $q=3$, we show that: (i) there is no $\mathcal{T}$-blocking set of size 10 , and (ii) there are exactly two $\mathcal{T}$-blocking sets of size 11 up to isomorphism. By means of the computer algebra systems GAP [13] and Sage [9], we find that there exist no $\mathcal{T}$-blocking sets of size $q^{2}+1$ for each odd prime power $q \leq 13$.
\end{abstract}

Keywords: Projective space, Blocking set, Conic, Ovoid, Hyperbolic quadric

AMS 2010 subject classification: 05B25, 51E21

\section{Introduction}

Throughout, $q$ is a prime power. Let $\operatorname{PG}(3, q)$ be the three dimensional projective space defined over a finite field of order $q$ and $Q^{+}(3, q)$ be a hyperbolic quadric in $\mathrm{PG}(3, q)$. One can refer to [6] for the basic properties of the points, lines and planes of $\operatorname{PG}(3, q)$ with respect to the quadric $Q^{+}(3, q)$. Every line of $\mathrm{PG}(3, q)$ meets $Q^{+}(3, q)$ in $0,1,2$ or $q+1$ points. We denote by $\mathcal{E}$ (respectively, $\left.\mathcal{T}_{1}, \mathcal{S}, \mathcal{T}_{0}\right)$ the set of lines of $\operatorname{PG}(3, q)$ that intersect $Q^{+}(3, q)$ in 0 (respectively, $\left.1,2, q+1\right)$ points. The elements of $\mathcal{E}$ are called external lines, those of $\mathcal{S}$ secant lines and those of $\mathcal{T}:=\mathcal{T}_{0} \cup \mathcal{T}_{1}$ tangent lines. If $L \in \mathcal{T}_{i}$ with $i \in\{0,1\}$, then $L$ is also called a $\mathcal{T}_{i}$-line. The $\mathcal{T}_{0}$-lines are precisely the lines contained in $Q^{+}(3, q)$, and so we have $\left|\mathcal{T}_{0}\right|=2(q+1)$. As every point of $Q^{+}(3, q)$ is contained in $q-1 \mathcal{T}_{1}$-lines, we have $\left|\mathcal{T}_{1}\right|=(q+1)^{2}(q-1)$ and hence $|\mathcal{T}|=(q+1)\left(q^{2}+1\right)$. We also have $|\mathcal{S}|=\frac{1}{2} q^{2}(q+1)^{2}$ and $|\mathcal{E}|=\left(q^{2}+1\right)\left(q^{2}+q+1\right)-(q+1)\left(q^{2}+1\right)-\frac{1}{2} q^{2}(q+1)^{2}=\frac{1}{2} q^{2}(q-1)^{2}$.

For a given nonempty set $\mathcal{L}$ of lines of $\mathrm{PG}(3, q)$, a set $X$ of points of $\mathrm{PG}(3, q)$ is called an $\mathcal{L}$-blocking set if each line of $\mathcal{L}$ meets $X$. The first step in the study of blocking sets has been to determine the smallest cardinality of a blocking set and to characterize, if possible, all blocking sets of that cardinality. If $\mathcal{L}$ is the set of all lines of $\operatorname{PG}(3, q)$ and $X$ 
is an $\mathcal{L}$-blocking set, then $|X| \geq q^{2}+q+1$ and equality holds if and only if $X$ is a plane of $\mathrm{PG}(3, q)$. This follows from a more general result by Bose and Burton [4, Theorem 1]. Biondi et al. characterized the minimum size $\mathcal{E}$-blocking sets in [2, Theorem 2.4] for $q \geq 9$ odd and in [1, Theorem 1.1] for $q \geq 8$ even (also see [10, Section 3] for a different proof which works for all even $q$ ). When $q>2$ is even, the minimum size $(\mathcal{E} \cup \mathcal{S})$-blocking sets were determined in [12, Theorem 1.3] using the properties of generalized quadrangles. For $\mathcal{L} \in\{\mathcal{S}, \mathcal{T} \cup \mathcal{S}, \mathcal{E} \cup \mathcal{S}\}$, the minimum size $\mathcal{L}$-blocking sets are described in [11] for all $q$. When $q$ is even, the minimum size $(\mathcal{E} \cup \mathcal{T})$-blocking sets are characterized in $[10$, Proposition 1.5].

Suppose $q$ is even and let $\zeta$ denote the symplectic polarity of $\mathrm{PG}(3, q)$ associated with the quadric $Q^{+}(3, q)$. With the symplectic polarity $\zeta$, there is associated a symplectic generalized quadrangle $W(q)$, whose points are the points of $\operatorname{PG}(3, q)$ and whose lines are the lines of $\mathrm{PG}(3, q)$ that are totally isotropic with respect to $\zeta$, with incidence being containment (see [8] for more on generalized quadrangles). The lines of $W(q)$ are precisely the elements of $\mathcal{T}$. If $X$ is a $\mathcal{T}$-blocking set in $\mathrm{PG}(3, q)$, then $|X| \geq q^{2}+1$ and equality holds if and only if $X$ is an ovoid ${ }^{1}$ of $W(q)$. There are two families of ovoids known, namely the classical ovoids (being elliptic quadrics of the ambient projective space PG(3,q)) and the Ree-Tits ovoids (which exist only when $q>2$ is a nonsquare).

In the $q$ odd case, nothing seemed to be known for the minimum size $\mathcal{T}$-blocking sets. If $k$ is the minimum size of such a blocking set, then the following bounds hold by Lemmas 2.1 and 2.2 in the next section:

$$
q^{2}+1 \leq k \leq q^{2}+q
$$

Calling two $\mathcal{T}$-blocking sets $X_{1}$ and $X_{2}$ isomorphic if there is an automorphism of $\mathrm{PG}(3, q)$ stabilizing $Q^{+}(3, q)$ and mapping $X_{1}$ to $X_{2}$, we prove the following (without the aid of a computer) for the case $q=3$.

Theorem 1.1. Suppose that $q=3$. Then there is no $\mathcal{T}$-blocking set of size 10 in $\mathrm{PG}(3,3)$. Up to isomorphism, there are two $\mathcal{T}$-blocking sets of size 11 in $\mathrm{PG}(3,3)$.

In Lemma 2.1 of the next section, we show that a $\mathcal{T}$-blocking set of size $q^{2}+1$ is an ovoid of the subgeometry of PG(3,q) defined by the tangent lines. In Section 4 of [3], computer code in Sage [9] can be found for classifying ovoids of point-line geometries. With the aid of this code and some computations in GAP [13], we were able to show the nonexistence of $\mathcal{T}$-blocking sets of size $q^{2}+1$ for certain small values of $q$, see [5].

Theorem 1.2. There exist no $\mathcal{T}$-blocking sets of size $q^{2}+1$ in $\operatorname{PG}(3, q)$ for each odd prime power $q \leq 13$.

In Section 2, we prove a few basic results. In Section 3, we construct two nonisomorphic $\mathcal{T}$-blocking sets in PG $(3,3)$ each of size 11 . Finally, in Section 4 , we prove the nonexistence of $\mathcal{T}$-blocking sets of size 10 and classify the $\mathcal{T}$-blocking sets of size 11 in $\mathrm{PG}(3,3)$.

\footnotetext{
${ }^{1} \mathrm{An}$ ovoid of a point-line geometry is a set of points meeting each line in a singleton.
} 
Acknowledgement: The first author would like to thank the National Institute of Science Education and Research, Bhubaneswar for the kind hospitality provided during his visit to the School of Mathematical Sciences in March-April 2017.

\section{Preliminaries}

As in Section 1, consider a hyperbolic quadric $Q^{+}(3, q)$ in $\operatorname{PG}(3, q)$. A lower bound for the sizes of $\mathcal{T}$-blocking sets is easily derived from the fact that there are $(q+1)\left(q^{2}+1\right)$ tangent lines in total and $q+1$ tangent lines through a given point.

Lemma 2.1. Let $X$ be a $\mathcal{T}$-blocking set in $\mathrm{PG}(3, q)$. Then $|X| \geq q^{2}+1$, with equality if and only if every tangent line contains a unique point of $X$.

Proof. Each of the $(q+1)\left(q^{2}+1\right)$ tangent lines contains at least one point of $X$. As every point of $\mathrm{PG}(3, q)$ is contained in precisely $q+1$ tangent lines, we have $|X| \geq \frac{(q+1)\left(q^{2}+1\right) \cdot 1}{q+1}=$ $q^{2}+1$. Equality holds if and only if every tangent line contains a unique point of $X$.

With the quadric $Q^{+}(3, q)$, there is naturally associated a polarity $\zeta$ which is symplectic if $q$ is even and orthogonal if $q$ is odd. For every point $x$ of $Q^{+}(3, q), x^{\zeta}$ is a plane which is tangent to $Q^{+}(3, q)$ at the point $x$ and intersects $Q^{+}(3, q)$ in the union of two lines through $x$. The $q+1$ tangent lines through $x$ are precisely the lines through $x$ contained in $x^{\zeta}$. By the following lemma, the size of a $\mathcal{T}$-blocking set is bounded above by $q^{2}+q$.

Lemma 2.2. Let $\pi$ be a plane of $\mathrm{PG}(3, q)$ which is tangent to $Q^{+}(3, q)$ at the point $x$. Then $\pi \backslash\{x\}$ is a $\mathcal{T}$-blocking set of size $q^{2}+q$.

Proof. We have $|\pi \backslash\{x\}|=q^{2}+q$. As every line meets $\pi$, every tangent line not containing $x$ meets $\pi \backslash\{x\}$. If $L$ is a tangent line containing $x$, then $L$ is contained in $x^{\zeta}=\pi$ and hence contains points of $\pi \backslash\{x\}$. So, $\pi \backslash\{x\}$ is a $\mathcal{T}$-blocking set.

Suppose $q$ is odd. For every point $x$ of $\mathrm{PG}(3, q) \backslash Q^{+}(3, q), x^{\zeta}$ is a nontangent plane with $x \notin x^{\zeta}$ and the set $O_{x}:=x^{\zeta} \cap Q^{+}(3, q)$ is a conic of $x^{\zeta}$. The $q+1$ tangent lines through $x$ are precisely the lines through $x$ meeting $O_{x}$. The conic $O_{x}$ is an ovoid of $Q^{+}(3, q)$, that is, a set of points intersecting each $\mathcal{T}_{0}$-line in a unique point. The map $x \mapsto O_{x}$ defines a bijection between $\mathrm{PG}(3, q) \backslash Q^{+}(3, q)$ and the set of conics contained in $Q^{+}(3, q)$. When $q=3$, we note that the set of conics contained in $Q^{+}(3,3)$ coincides with the set of ovoids of $Q^{+}(3,3)$. If $x \in \mathrm{PG}(3, q) \backslash Q^{+}(3, q)$, then the number of secant lines through $x$ is equal to $\frac{\left|Q^{+}(3, q) \backslash O_{x}\right|}{2}=\frac{(q+1) q}{2}$ and the number of external lines through $x$ is equal to $\left(q^{2}+q+1\right)-(q+1)-\frac{(q+1) q}{2}=\frac{(q-1) q}{2}$.

Since $q$ is odd, every point of $x^{\zeta} \backslash O_{x}$ lies on 0 or $2 \mathcal{T}_{1}$-lines contained in $x^{\zeta}$. Such a point is called interior to $O_{x}$ in the first case and exterior to $O_{x}$ in the latter. There are $q(q-1) / 2$ interior points and $q(q+1) / 2$ exterior points in $x^{\zeta}$ with respect to $O_{x}$. Every interior point lies on $(q+1) / 2$ external lines and $(q+1) / 2$ secant lines contained in $x^{\zeta}$. Every exterior point lies on $(q-1) / 2$ external lines and $(q-1) / 2$ secant lines contained 
in $x^{\zeta}$. Every external line contained in $x^{\zeta}$ contains $(q+1) / 2$ interior points and $(q+1) / 2$ exterior points. Every secant line contained in $x^{\zeta}$ contains $(q-1) / 2$ interior points and $(q-1) / 2$ exterior points. One can refer to [7] for these basic properties.

Lemma 2.3. Suppose $x \in \mathrm{PG}(3, q) \backslash Q^{+}(3, q)$ with $q$ odd. Then each line of $\mathrm{PG}(3, q)$ through $x$, which is external to $Q^{+}(3, q)$, meets $x^{\zeta}$ in a point interior to $O_{x}$.

Proof. Let $L$ be an external line through $x$. Since $x \notin x^{\zeta}, L$ contains exactly one point of $x^{\zeta}$. Denote this point by $z$. We show that $z$ is interior to $O_{x}$.

Suppose this is not true. Then $z$ is exterior to $O_{x}$. Let $M$ be a $\mathcal{T}_{1}$-line through $z$ in $x^{\zeta}$ and $\pi$ be the plane generated by $L$ and $M$. Then $\pi$ is a nontangent plane, as it contains the external line $L$. On the other hand, if $y$ is the unique point of the intersection $M \cap O_{x}$, then the $\mathcal{T}_{1}$-line $M_{1}:=x y$ is contained in $\pi$. So $\pi$ is also the plane generated by the tangent lines $M$ and $M_{1}$. It follows that $\pi$ is the plane which is tangent to $Q^{+}(3, q)$ at the point $y$, a contradiction.

Again under the assumption that $x \in \mathrm{PG}(3, q) \backslash Q^{+}(3, q)$ with $q$ odd, we denote by $\mathcal{E}_{x}$ the set of lines in $\mathrm{PG}(3, q)$ through $x$ that are external to $Q^{+}(3, q)$, and by $I_{x}$ the set of interior points in $x^{\zeta}$ with respect to the conic $O_{x}$. We have $\left|\mathcal{E}_{x}\right|=q(q-1) / 2=\left|I_{x}\right|$. As a consequence of Lemma 2.3, we have the following.

Corollary 2.4. Suppose $x \in \mathrm{PG}(3, q) \backslash Q^{+}(3, q)$ with $q$ odd. Then the map from $\mathcal{E}_{x}$ to $I_{x}$, sending each line in $\mathcal{E}_{x}$ to its point of intersection with $I_{x}$, is bijective.

Proof. By Lemma 2.3, the map is well-defined and is injective. Since $\left|\mathcal{E}_{x}\right|=\left|I_{x}\right|$, the map is surjective also.

In the special case that $q=3$, the following can be said.

Lemma 2.5. Suppose $q=3$. Let $\pi_{1}$ be a nontangent plane and $O_{1}$ be the conic $\pi_{1} \cap$ $Q^{+}(3,3)$ in $\pi_{1}$. Fix a line $L$ in $\pi_{1}$ which is external to $O_{1}$. Then there exists exactly one more nontangent plane $\pi_{2}$ satisfying the following:

(1) $L$ is an external line in $\pi_{2}$ with respect to the conic $O_{2}:=\pi_{2} \cap Q^{+}(3,3)$.

(2) If $a \in L$ is exterior (respectively, interior) to $O_{1}$ in $\pi_{1}$, then it is also exterior (respectively, interior) to $\mathrm{O}_{2}$ in $\pi_{2}$.

In fact, if $a \in L$ is exterior to $O_{1}$ in $\pi_{1}$, then the two $\mathcal{T}_{1}$-lines through a not in $\pi_{1}$ are contained in $\pi_{2}$.

Proof. Let $x$ be the point in $\mathrm{PG}(3,3) \backslash Q^{+}(3,3)$ such that $O_{x}=O_{1}$. Such a point $x$ exists, since the map $\alpha \mapsto O_{\alpha}:=\alpha^{\zeta} \cap Q^{+}(3,3)$ is a bijection between $\mathrm{PG}(3,3) \backslash Q^{+}(3,3)$ and the set of conics contained in $Q^{+}(3,3)$. We have $\pi_{1}=x^{\zeta}$. Write $L=\left\{a, b, z_{1}, z_{2}\right\}$, where $a, b$ (respectively, $z_{1}, z_{2}$ ) are exterior (respectively, interior) to $O_{1}$ in $\pi_{1}$. By Corollary 2.4, the lines $T_{1}:=x z_{1}$ and $T_{2}:=x z_{2}$ are external lines.

Let $\pi_{2}$ be the plane generated by the line $L$ and the point $x$. Then $\pi_{2}$ is a nontangent plane in which $L$ is external to the conic $O_{2}:=\pi_{2} \cap Q^{+}(3,3)$. The lines $T_{1}$ and $T_{2}$ in $\pi_{2}$ 
are external to $O_{2}$. Thus, for $i \in\{1,2\}, L$ and $T_{i}$ are two external lines in $\pi_{2}$ through $z_{i}$. It follows that both $z_{1}$ and $z_{2}$ are interior to $O_{2}$ in $\pi_{2}$. This implies that both $a$ and $b$ must be exterior to $\mathrm{O}_{2}$ in $\pi_{2}$. Hence $\pi_{2}$ satisfies the conditions (1) and (2).

Out of the four $\mathcal{T}_{1}$-lines through $a$ (respectively, through $b$ ), two are contained in $\pi_{1}$ and the other two are in $\pi_{2}$ (as $\pi_{1} \cap \pi_{2}=L$ is not a $\mathcal{T}_{1}$-line). This must hold for any nontangent plane satisfying the conditions (1) and (2). This fact implies the uniqueness of $\pi_{2}$ satisfying (1) and (2).

\section{Two constructions of $\mathcal{T}$-blocking sets}

In this section, we construct two nonisomorphic $\mathcal{T}$-blocking sets of size 11 each in $\mathrm{PG}(3,3)$.

\subsection{First construction}

Consider a point $x \in \mathrm{PG}(3,3) \backslash Q^{+}(3,3)$ and let $I_{x}=\left\{z_{1}, z_{2}, z_{3}\right\}$. Fix a line $L$ in the plane $x^{\zeta}$ which is external to $O_{x}$. Then $L$ contains exactly two points of $I_{x}$, say $z_{2}$ and $z_{3}$. Let $\bar{L}$ be the unique line in $\mathcal{E}_{x}$ such that $\bar{L}$ meets $x^{\zeta}$ in $z_{1}$, see Corollary 2.4. Define the following set:

$$
B_{1}:=O_{x} \cup L \cup(\bar{L} \backslash\{x\}) .
$$

We prove the following.

Proposition 3.1. $B_{1}$ is a $\mathcal{T}$-blocking set of size 11 in $\mathrm{PG}(3,3)$.

Proof. Clearly, $\left|B_{1}\right|=11$. Let $A=x^{\zeta} \backslash B_{1}$. Then $A$ consists of four exterior points, each of which is different from the two exterior points contained in $L$. Since every tangent line meets $x^{\zeta}$, it is enough to prove that each $\mathcal{T}_{1}$-line through a point of $A$ meets $B_{1}$.

Take a point $a \in A$ and a $\mathcal{T}_{1}$-line $T$ through $a$. If $T$ is contained in $x^{\zeta}$, then observe that $T$ meets $B_{1}$ in two points, one from $O_{x}$ and the other one is an exterior point contained in $L$. So assume that $T$ is not contained in $x^{\zeta}$. We show that $T$ contains a point of $B_{1} \backslash x^{\zeta}=\bar{L} \backslash\left\{x, z_{1}\right\}$.

Let $M$ be the line in $x^{\zeta}$ through $a$ and $z_{1}$. Then $M$ is either external or secant to $O_{x}$ in $x^{\zeta}$, as it contains the interior point $z_{1}$. Since $M$ has to intersect the external line $L$ in $x^{\zeta}$ in a point different from $a$ and $z_{1}$, it follows that $M$ can not be secant to $O_{x}$. So $M$ is external to $O_{x}$ in $x^{\zeta}$ and hence contains an interior point different from $z_{1}$. Without loss, we may assume that $M$ contains $z_{2}$ as the second interior point.

Setting $\pi_{1}=x^{\zeta}$ and taking the external line $M$ of $\pi_{1}$ in Lemma 2.5, we get a nontangent plane $\pi_{2}$ containing $M$ such that $z_{1}, z_{2}$ are interior points and $a$ is an exterior point in $\pi_{2}$ with respect to the conic $O_{2}:=\pi_{2} \cap Q^{+}(3,3)$. Note that $T$ is a $\mathcal{T}_{1}$-line through $a$ in $\pi_{2}$.

Let $\bar{M}(\neq M)$ be the second line in $\pi_{2}$ through $z_{1}$ which is external to $O_{2}$. Out of the three lines through $z_{1}$ external to $Q^{+}(3,3)$, the line $M$ is common to both the planes $\pi_{1}=x^{\zeta}$ and $\pi_{2}$. The plane $x^{\zeta}$ contains one more external line through $z_{1}$. So $\bar{M}$ must be the external line through $x$ which corresponds to the point $z_{1}$ under the map defined in Corollary 2.4. It follows that $\bar{M}=\bar{L}$. As $x z_{1}$ and $x z_{2}$ are external lines in $\pi_{2}$ (by 
Corollary 2.4), $x$ must be interior to $O_{2}$ in $\pi_{2}$. Since the $\mathcal{T}_{1}$-line $T$ and the external line $\bar{L}$ in $\pi_{2}$ meet in a point exterior to $O_{2}$, it follows that $T$ contains a point of $\bar{L} \backslash\left\{x, z_{1}\right\}$. This completes the proof.

\subsection{Second construction}

Fix a point $x \in \mathrm{PG}(3,3) \backslash Q^{+}(3,3)$ and let $I_{x}=\left\{z_{1}, z_{2}, z_{3}\right\}$. Let $y$ be a point in $x^{\zeta}$ exterior to $O_{x}$. Let $L_{1}$ and $L_{2}$ be the two $\mathcal{T}_{1}$-lines through $y$ which are not contained in $x^{\zeta}$. For $i \in\{1,2\}$, let $w_{i}$ be the tangency point of $L_{i}$ in $Q^{+}(3,3)$. Define the following set:

$$
B_{2}:=O_{x} \cup I_{x} \cup\left(L_{1} \backslash\left\{y, w_{1}\right\}\right) \cup\left(L_{2} \backslash\left\{y, w_{2}\right\}\right) .
$$

We prove the following:

Proposition 3.2. $B_{2}$ is a $\mathcal{T}$-blocking set of size 11 in $\mathrm{PG}(3,3)$.

Proof. Clearly, $\left|B_{2}\right|=11$. Let $D=x^{\zeta} \backslash B_{2}$. Then $D$ consists of the six exterior points in $x^{\zeta}$ with respect to $O_{x}$. Since every tangent line meets $x^{\zeta}$, it is enough to prove that each $\mathcal{T}_{1}$-line through a point of $D$ meets $B_{2}$.

Take a point $a \in D$ and a $\mathcal{T}_{1}$-line $T$ through $a$. If $T$ is contained in $x^{\zeta}$, then $T$ meets $B_{2}$ in the unique point of $T \cap O_{x}$. So assume that $T$ is not contained in $x^{\zeta}$. If $a=y$, then $T$ is either $L_{1}$ or $L_{2}$ and hence meets $B_{2}$ at two points. Assume that $a \neq y$. Since both $a$ and $y$ are exterior to $O_{x}$, the line $M:=a y$ in $x^{\zeta}$ is either tangent or external to $O_{x}$.

Case I: $M$ is tangent to $O_{x}$. Let $\pi$ be the nontangent plane generated by the lines $T$ and $M$. Denote by $O_{\pi}$ the conic $\pi \cap Q^{+}(3,3)$ in $\pi$. The point $y$ in $\pi$ is exterior to $O_{\pi}$. So there exists one more $\mathcal{T}_{1}$-line in $\pi$ (different from $M$ ) containing $y$. Since $\pi \cap x^{\zeta}=M$, it follows that either $L_{1}$ or $L_{2}$ is a line in $\pi$. Without loss, we may assume that $L_{1}$ is a line in $\pi$. The lines $T$ and $L_{1}$ intersect in $\pi$ in a point different from $y$ and $w_{1}$. So $T$ meets $B_{2}$ at a point of $L_{1} \backslash\left\{y, w_{1}\right\}$.

Case II: $M$ is external to $O_{x}$. Setting $\pi_{1}=x^{\zeta}$ and taking the external line $M$ of $\pi_{1}$ in Lemma 2.5, we get a nontangent plane $\pi_{2}$ through $M$ containing the lines $T, L_{1}$ and $L_{2}$. Now it can be seen that $T$ intersects $L_{1}$ (respectively, $L_{2}$ ) in $\pi_{2}$ at a point different from $y$ and $w_{1}$ (respectively, $w_{2}$ ). So $T$ meets $B_{2}$ at two points, one from $L_{1} \backslash\left\{y, w_{1}\right\}$ and one from $L_{2} \backslash\left\{y, w_{2}\right\}$.

Thus $B_{2}$ is a $\mathcal{T}$-blocking set of $\mathrm{PG}(3,3)$ of size 11 . This completes the proof.

\subsection{The blocking sets $B_{1}$ and $B_{2}$ are nonisomorphic}

Proposition 3.3. The two blocking sets $B_{1}$ and $B_{2}$ are nonisomorphic.

Proof. Write $B_{2}$ as a disjoint union $B_{2}=\left(B_{2} \cap x^{\zeta}\right) \cup\left(B_{2} \backslash x^{\zeta}\right)$. Observe that any line meets $B_{2} \backslash x^{\zeta}$ in at most two points. Let $R$ be a line external to $Q^{+}(3,3)$. If $R$ is a line in $x^{\zeta}$, then $R$ meets $B_{2}$ at exactly two points of $B_{2} \cap x^{\zeta}$ (which come from $I_{x}$ ) and is disjoint from $B_{2} \backslash x^{\zeta}$. Suppose that $R$ is not a line in $x^{\zeta}$. Then $R$ contains at most one point from 
$B_{2} \cap x^{\zeta}$ and at most two points from $B_{2} \backslash x^{\zeta}$. So $R$ is not contained in $B_{2}$. Thus every external line meets $B_{2}$ in at most three points.

However, from the construction of $B_{1}$, it is clear that $B_{1}$ contains a line external to $Q^{+}(3,3)$. So $B_{1}$ and $B_{2}$ are nonisomorphic.

\section{$4 \mathcal{T}$-blocking sets of sizes $\mathbf{1 0}$ and $\mathbf{1 1}$ in $\mathrm{PG}(3,3)$}

Consider a hyperbolic quadric $Q^{+}(3,3)$ in $\mathrm{PG}(3,3)$. We label the points of $Q^{+}(3,3)$ by $x_{i j}$ where $i, j \in\{1,2,3,4\}$ such that two distinct points $x_{i j}$ and $x_{i^{\prime} j^{\prime}}$ of $Q^{+}(3,3)$ are incident with a $\mathcal{T}_{0}$-line if either $i=i^{\prime}$ or $j=j^{\prime}$.

We denote by $O^{*}$ the ovoid $\left\{x_{11}, x_{22}, x_{33}, x_{44}\right\}$ of $Q^{+}(3,3)$. There are nine ovoids of $Q^{+}(3,3)$ that are disjoint from $O^{*}$. These are:

$$
\begin{aligned}
& O_{1}=\left\{x_{12}, x_{21}, x_{34}, x_{43}\right\}, O_{2}=\left\{x_{13}, x_{31}, x_{24}, x_{42}\right\}, O_{3}=\left\{x_{14}, x_{41}, x_{23}, x_{32}\right\}, \\
& O_{4}=\left\{x_{12}, x_{24}, x_{43}, x_{31}\right\}, O_{5}=\left\{x_{12}, x_{23}, x_{34}, x_{41}\right\}, O_{6}=\left\{x_{13}, x_{24}, x_{32}, x_{41}\right\}, \\
& O_{7}=\left\{x_{13}, x_{21}, x_{34}, x_{42}\right\}, O_{8}=\left\{x_{14}, x_{21}, x_{32}, x_{43}\right\}, O_{9}=\left\{x_{14}, x_{23}, x_{31}, x_{42}\right\} .
\end{aligned}
$$

Lemma 4.1. There are four collections, each of six ovoids from $\left\{O_{1}, O_{2}, \ldots, O_{9}\right\}$, such that every point of $Q^{+}(3,3) \backslash O^{*}$ is contained in precisely two ovoids of a given collection. These four collections are $\mathcal{C}^{*}=\left\{O_{4}, O_{5}, O_{6}, O_{7}, O_{8}, O_{9}\right\},\left\{O_{1}, O_{2}, O_{5}, O_{6}, O_{8}, O_{9}\right\}$, $\left\{O_{1}, O_{3}, O_{4}, O_{6}, O_{7}, O_{9}\right\}$ and $\left\{O_{2}, O_{3}, O_{4}, O_{5}, O_{7}, O_{8}\right\}$.

Proof. It is easily verified that each of these four collections satisfies the required condition. Conversely, suppose that $\mathcal{C} \neq \mathcal{C}^{*}$ is a collection of six ovoids satisfying the condition of the lemma. As $\mathcal{C} \neq \mathcal{C}^{*}$, at least one of $O_{1}, O_{2}, O_{3}$ is contained in $\mathcal{C}$. Now, any partition of $Q^{+}(3,3) \backslash O^{*}$ in three ovoids must contain either one or three ovoids of the set $\left\{O_{1}, O_{2}, O_{3}\right\}$, implying that at least one of $O_{1}, O_{2}, O_{3}$ is not contained in $\mathcal{C}$.

Suppose $O_{1} \in \mathcal{C}$ and $O_{2} \notin \mathcal{C}$. As each of $x_{13}, x_{31}$ should be contained in two ovoids of $\mathcal{C}$, we then must have $O_{4}, O_{6}, O_{7}, O_{9} \in \mathcal{C}$. At this stage, $x_{12}$ and $x_{21}$ are already contained in two ovoids of the collection $\mathcal{C}$, implying that $O_{5}$ and $O_{8}$ do not belong to $\mathcal{C}$. So, $\mathcal{C}$ is necessarily equal to $\left\{\mathrm{O}_{1}, \mathrm{O}_{3}, \mathrm{O}_{4}, \mathrm{O}_{6}, \mathrm{O}_{7}, \mathrm{O}_{9}\right\}$.

By symmetry we then see that $\mathcal{C}$ always contains precisely two ovoids of the set $\left\{O_{1}, O_{2}, O_{3}\right\}$. If $O_{1}, O_{2} \in \mathcal{C}$ and $O_{3} \notin \mathcal{C}$, then a similar reasoning as above shows that $\mathcal{C}=\left\{O_{1}, O_{2}, O_{5}, O_{6}, O_{8}, O_{9}\right\}$. Similarly, if $O_{2}, O_{3} \in \mathcal{C}$ and $O_{1} \notin \mathcal{C}$, then $\mathcal{C}=$ $\left\{\mathrm{O}_{2}, \mathrm{O}_{3}, \mathrm{O}_{4}, \mathrm{O}_{5}, \mathrm{O}_{7}, \mathrm{O}_{8}\right\}$.

Invoking Lemma 4.1, the verification of the following lemma is straightforward.

Lemma 4.2. Suppose $\mathcal{C}$ is a collection of six ovoids from $\left\{O_{1}, O_{2}, \ldots, O_{9}\right\}$ such that every point of $Q^{+}(3,3) \backslash O^{*}$ is contained in precisely two ovoids of $\mathcal{C}$. Let $S$ denote the set of all points $x \in Q^{+}(3,3) \backslash O^{*}$ such that $\{x\}$ is the intersection of two distinct ovoids of $\mathcal{C}$. Then $S=Q^{+}(3,3) \backslash O^{*}$ if $\mathcal{C}=\mathcal{C}^{*}$, and $S=O$ if $\mathcal{C} \neq \mathcal{C}^{*}$, where $O$ is the unique element of $\left\{\mathrm{O}_{1}, \mathrm{O}_{2}, \mathrm{O}_{3}\right\}$ not contained in $\mathcal{C}$. 
Lemma 4.3. Let $x$ be a point of $Q^{+}(3,3)$ and let $L_{1}=\left\{x, y_{1}, y_{2}, y_{3}\right\}$ and $L_{2}=\left\{x, z_{1}, z_{2}, z_{3}\right\}$ be the two $\mathcal{T}_{1}$-lines through $x$. Then the following hold:

(1) $\left\{O_{y_{1}}, O_{y_{2}}, O_{y_{3}}\right\}$ (resp. $\left.\left\{O_{z_{1}}, O_{z_{2}}, O_{z_{3}}\right\}\right)$ is a set of ovoids of $Q^{+}(3,3)$ through $x$ partitioning the set of points of $Q^{+}(3,3)$ noncollinear with $x$.

(2) If $i, j \in\{1,2,3\}$, then $O_{y_{i}} \cap O_{z_{j}}$ contains precisely two points (one of which is $x$ ).

Proof. (1) As $L_{1}$ is a $\mathcal{T}_{1}$-line, we see that $x \in O_{y_{i}}$ for every $i \in\{1,2,3\}$. Now, take an arbitrary point $u \in Q^{+}(3,3)$ noncollinear with $x$. Then $u^{\zeta}$ does not contain $x$ and so intersects $L_{1}$ in a unique point $y_{i}$. The point $y_{i}$ is the unique point $v$ of $L_{1} \backslash\{x\}$ for which $u \in v^{\zeta}$. So, $\left\{O_{y_{1}}, O_{y_{2}}, O_{y_{3}}\right\}$ partitions the set of points of $Q^{+}(3,3)$ noncollinear with $x$. A similar argument holds for the line $L_{2}$.

(2) There are six ovoids through the point $x$. One coincides with $O_{y_{i}}$, two $\left(O_{y_{r}}, O_{y_{s}}\right)$ intersect $O_{y_{i}}$ in $\{x\}$ where $\{i, r, s\}=\{1,2,3\}$, and the remaining three (necessarily $O_{z_{1}}$, $O_{z_{2}}, O_{z_{3}}$ ) intersect $O_{y_{i}}$ in two points (one of which is $x$ ).

\subsection{Nonexistence of $\mathcal{T}$-blocking sets of size 10}

The following result proves the nonexistence of $\mathcal{T}$-blocking sets of size 10 in $\mathrm{PG}(3,3)$.

Proposition 4.4. There are no $\mathcal{T}$-blocking sets of size 10 in $\mathrm{PG}(3,3)$.

Proof. Suppose $X$ is a $\mathcal{T}$-blocking sets of size 10 in PG(3,3). By Lemma 2.1, we then know that each tangent line contains a unique point of $X$. In particular, $O:=X \cap Q^{+}(3,3)$ is an ovoid of $Q^{+}(3,3)$ and $Y:=X \backslash Q^{+}(3,3)$ is a set of 6 points outside $Q^{+}(3,3)$ intersecting each $\mathcal{T}_{1}$-line in a unique point. Without loss of generality, we may suppose that $O=O^{*}$. We show the following properties for the collection $\mathcal{C}=\left\{O_{y} \mid y \in Y\right\}$ :

(a) all ovoids of $\mathcal{C}$ are disjoint from $O$;

(b) any two ovoids of $\mathcal{C}$ cannot intersect in a singleton;

(c) every point of $Q^{+}(3,3) \backslash O$ is contained in precisely two ovoids of $\mathcal{C}$.

If $O_{y}$ with $y \in Y$ contains a point $x \in O$, then the tangent line $x y$ would contain two points of $X=O \cup Y$, namely $x$ and $y$, a contradiction. If $O_{y_{1}} \cap O_{y_{2}}$ is a singleton $\{x\}$, where $y_{1}, y_{2} \in Y$ with $y_{1} \neq y_{2}$, then Lemma 4.3 would imply that there is a $\mathcal{T}_{1}$-line through $x$ containing $y_{1}$ and $y_{2}$, a contradiction. Finally, every point $x \in Q^{+}(3,3) \backslash O$ is contained in two $\mathcal{T}_{1}$-lines, each containing exactly one point of $Y$, showing that $x$ is contained in precisely two ovoids of $\mathcal{C}$.

By Lemmas 4.1 and 4.2, we however know that there are no collections $\mathcal{C}$ of six ovoids that satisfy the above properties (a), (b) and (c). 


\subsection{Classification of the $\mathcal{T}$-blocking sets of size 11}

In the rest of the paper, we classify the $\mathcal{T}$-blocking sets of size 11 in $\operatorname{PG}(3,3)$. We show that there are only two such $\mathcal{T}$-blocking sets up to isomorphism, necessarily isomorphic to the blocking sets $B_{1}$ and $B_{2}$ constructed in Section 3 .

Lemma 4.5. If $X$ is a $\mathcal{T}$-blocking set of size 11 in $\mathrm{PG}(3,3)$, then $\left|X \backslash Q^{+}(3,3)\right| \in\{6,7\}$ and $\left|X \cap Q^{+}(3,3)\right| \in\{4,5\}$.

Proof. Since $\left|X \cap Q^{+}(3,3)\right| \leq|X|<12$, there exists a line $L$ in $Q^{+}(3,3)$ meeting $X$ in either 1 or 2 points. Suppose every line of $Q^{+}(3,3)$ meets $X$ in 2 points. Then $\left|X \cap Q^{+}(3,3)\right|=8$. If $L$ is a line of $Q^{+}(3,3)$ and $L \backslash X=\{a, b\}$, then each of the four $\mathcal{T}_{1}$-lines meeting $\{a, b\}$ contains at least one point of $X \backslash Q^{+}(3,3)$. Any collection of four points of $X \backslash Q^{+}(3,3)$ that arise in this way are mutually distinct, implying that $|X|=\left|X \cap Q^{+}(3,3)\right|+\left|X \backslash Q^{+}(3,3)\right| \geq 8+4=12$, which is a contradiction.

Hence, there exists a line $L$ in $Q^{+}(3,3)$ meeting $X$ in a unique point. If $L \backslash X=\{a, b, c\}$, then there are six $\mathcal{T}_{1}$-lines meeting $\{a, b, c\}$ and each of these six $\mathcal{T}_{1}$-lines contains at least one point of $X \backslash Q^{+}(3,3)$. Any collection of six points of $X \backslash Q^{+}(3,3)$ that arise in this way are mutually distinct, implying that $\left|X \backslash Q^{+}(3,3)\right| \geq 6$. As $\left|X \cap Q^{+}(3,3)\right| \geq 4$, we thus have that $\left|X \backslash Q^{+}(3,3)\right| \in\{6,7\}$ and $\left|X \cap Q^{+}(3,3)\right| \in\{4,5\}$.

Proposition 4.6. If $X$ is a $\mathcal{T}$-blocking set of size 11 in $\mathrm{PG}(3,3)$, then $\left|X \cap Q^{+}(3,3)\right|=4$ and $\left|X \backslash Q^{+}(3,3)\right|=7$.

Proof. Suppose that this is not the case. Then $\left|X \cap Q^{+}(3,3)\right|=5$ and $\left|X \backslash Q^{+}(3,3)\right|=6$ by Lemma 4.5. As each $\mathcal{T}_{0}$-line contains a point of $X$, there are precisely two $\mathcal{T}_{0}$-lines $L_{1}$ and $L_{2}$ that contain exactly two points of $X$ (while every other $\mathcal{T}_{0}$-line intersects $X$ in a singleton). The lines $L_{1}$ and $L_{2}$ belong to distinct parallel classes of lines of $Q^{+}(3,3)$. We distinguish two cases.

Case I. The singleton $L_{1} \cap L_{2}$ is not contained in $X$. Without loss of generality, we may suppose that $X \cap Q^{+}(3,3)=\left\{x_{12}, x_{13}, x_{21}, x_{31}, x_{44}\right\}$. The reasoning in Lemma 4.5 leading to the inequality $\left|X \backslash Q^{+}(3,3)\right| \geq 6$ shows that if $L$ is a $\mathcal{T}_{0}$-line meeting $X$ in a singleton, then any $\mathcal{T}_{1}$-line meeting $L \backslash X$ cannot contain more than one point of $X$, and any $\mathcal{T}_{1}$-line meeting $L \cap X$ cannot contain a point of $X \backslash Q^{+}(3,3)$. As any point of $Q^{+}(3,3) \backslash\left\{x_{11}\right\}$ is contained in a $\mathcal{T}_{0}$-line intersecting $X$ in a singleton, we thus see from Lemma 4.3 that any two ovoids $O_{y_{1}}$ and $O_{y_{2}}$, where $y_{1}, y_{2} \in X \backslash Q^{+}(3,3)$, cannot intersect in a singleton distinct from $\left\{x_{11}\right\}$. Also, no ovoid $O_{y}$ with $y \in X \backslash Q^{+}(3,3)$ can contain a point of $X \cap Q^{+}(3,3)$. It can be seen that there are exactly six ovoids of $Q^{+}(3,3)$ disjoint from $X \cap Q^{+}(3,3)$ and so these ovoids are precisely the six ovoids $O_{y}$, where $y \in X \backslash Q^{+}(3,3)$. But that is impossible as two of these ovoids, namely $\left\{x_{11}, x_{23}, x_{34}, x_{42}\right\}$ and $\left\{x_{14}, x_{23}, x_{32}, x_{41}\right\}$, intersect in the singleton $\left\{x_{23}\right\} \neq\left\{x_{11}\right\}$.

Case II. The singleton $L_{1} \cap L_{2}$ is contained in $X$. Without loss of generality, we may suppose that $X \cap Q^{+}(3,3)=O^{*} \cup\left\{x_{12}\right\}$. The reasoning in Lemma 4.5 leading to the inequality $\left|X \backslash Q^{+}(3,3)\right| \geq 6$ shows that if $L$ is a $\mathcal{T}_{0}$-line meeting $Q^{+}(3,3) \cap X$ in a 
singleton, then each of the $\mathcal{T}_{1}$-lines meeting $L \backslash X$ cannot contain more than one point of $X$. As any point of $Q^{+}(3,3) \backslash\left\{x_{12}\right\}$ is contained in a line of $Q^{+}(3,3)$ intersecting $X$ in a singleton, we thus see from Lemma 4.3 that any two ovoids $O_{y_{1}}$ and $O_{y_{2}}$, where $y_{1}, y_{2} \in X \backslash Q^{+}(3,3)$, cannot intersect in a singleton distinct from $\left\{x_{12}\right\}$.

Put $\mathcal{C}=\left\{O_{y} \mid y \in X \backslash Q^{+}(3,3)\right\}$. Then $\mathcal{C}$ is a set of six ovoids of $Q^{+}(3,3)$, no two of which intersect in a singleton distinct from $\left\{x_{12}\right\}$. Moreover, each point $x \in Q^{+}(3,3) \backslash X$ is contained in precisely two $\mathcal{T}_{1}$-lines and hence in precisely two ovoids of $\mathcal{C}$.

We count the number of pairs $(L, x)$, where $L$ is a $\mathcal{T}_{1}$-line disjoint from $X \cap Q^{+}(3,3)$ and $x \in L \cap X$. There are $\left|Q^{+}(3,3) \backslash X\right| \cdot 2=22$ possibilities for $L$, and each such $L$ contains a unique point of $X$, implying that there are 22 such pairs. On the other hand, there are 6 possibilities for $x \in X \backslash Q^{+}(3,3)$.

Since $6 \cdot 3=18$, there are at least $22-18=4$ points of $X \backslash Q^{+}(3,3)$ whose induced ovoids are disjoint from $Q^{+}(3,3) \cap X$. There are six ovoids of $Q^{+}(3,3)$ that are disjoint from $X \cap Q^{+}(3,3)$ :

$$
\begin{array}{lll}
A_{1}=\left\{x_{13}, x_{24}, x_{31}, x_{42}\right\}, & A_{2}=\left\{x_{14}, x_{23}, x_{32}, x_{41}\right\}, & A_{3}=\left\{x_{13}, x_{21}, x_{34}, x_{42}\right\}, \\
A_{4}=\left\{x_{13}, x_{24}, x_{32}, x_{41}\right\}, & A_{5}=\left\{x_{14}, x_{23}, x_{31}, x_{42}\right\}, & A_{6}=\left\{x_{14}, x_{21}, x_{32}, x_{43}\right\} .
\end{array}
$$

Among the six ovoids that we have to choose for the set $\mathcal{C}$, at least four come from the collection $\left\{A_{1}, A_{2}, \ldots, A_{6}\right\}$. As exactly two of the six ovoids of $\mathcal{C}$ contain $x_{13}$, at most two of $A_{1}, A_{3}, A_{4}$ can occur in $\mathcal{C}$. Similarly, by considering the point $x_{14}$, we see that at most two of $A_{2}, A_{5}, A_{6}$ can occur in $\mathcal{C}$. We can conclude that precisely two of $A_{1}, A_{3}, A_{4}$, as well as precisely two of $A_{2}, A_{5}, A_{6}$ belong to $\mathcal{C}$. As $A_{3} \cap A_{4}$ and $A_{5} \cap A_{6}$ are singletons distinct from $\left\{x_{12}\right\}$, the ovoids $A_{1}$ and $A_{2}$ must belong to $\mathcal{C}$. Then the fact that $A_{3} \cap A_{5}$, $A_{3} \cap A_{6}$ and $A_{4} \cap A_{6}$ are singletons distinct from $\left\{x_{12}\right\}$ implies that $A_{3}$ and $A_{6}$ cannot belong to $\mathcal{C}$. So, $\mathcal{C}$ certainly contains the ovoids $A_{1}, A_{2}, A_{4}$ and $A_{5}$.

We still need to find two additional ovoids for $\mathcal{C}$. As the points $x_{21}, x_{34}$ and $x_{43}$ are not contained in $A_{1} \cup A_{2} \cup A_{4} \cup A_{5}$ and need to be covered twice, each of these two ovoids should contain these points. But that is impossible as there is only one ovoid containing these three points, namely $\left\{x_{12}, x_{21}, x_{34}, x_{43}\right\}$.

In the sequel, we suppose that $X$ is a set of 11 points of $\mathrm{PG}(3,3)$ that is a $\mathcal{T}$-blocking set. Then $\left|X \cap Q^{+}(3,3)\right|=4$ and $\left|X \backslash Q^{+}(3,3)\right|=7$ by Proposition 4.6. In fact, $U_{1}:=X \cap Q^{+}(3,3)$ is an ovoid of $Q^{+}(3,3)$. Denote by $U_{2}$ the subset of $Q^{+}(3,3)$ consisting of the following points:

- points of $X \cap Q^{+}(3,3)$ contained in a $\mathcal{T}_{1}$-line that contains points of $X \backslash Q^{+}(3,3)$,

- points of $Q^{+}(3,3) \backslash X$ contained in a $\mathcal{T}_{1}$-line that contains at least two points of $X \backslash Q^{+}(3,3)$.

Lemma 4.7. The set $U_{2}$ is an ovoid of $Q^{+}(3,3)$.

Proof. Let $L$ be a line of $Q^{+}(3,3)$ and put $\left\{x_{L}\right\}:=L \cap U_{1}$. For every $y \in X \backslash Q^{+}(3,3)$ denote by $y^{\prime}$ the unique point of $L \cap O_{y}$, that is, the unique point $y^{\prime}$ of $L$ for which $y y^{\prime}$ is 
a $\mathcal{T}_{1}$-line. Each $\mathcal{T}_{1}$-line meeting $L \backslash\left\{x_{L}\right\}$ contains at least one point of $X \backslash Q^{+}(3,3)$, and so each point of $L \backslash\left\{x_{L}\right\}$ is the image of at least two points of $X \backslash Q^{+}(3,3)$ under the map $y \mapsto y^{\prime}$. So, precisely one of the following two cases occurs:

(a) The point $x_{L}$ is the image of precisely one point of $X \backslash Q^{+}(3,3)$ and each of the three points of $L \backslash\left\{x_{L}\right\}$ is the image of precisely two points of $X \backslash Q^{+}(3,3)$.

(b) There exists a unique point $x_{L}^{\prime}$ on $L \backslash\left\{x_{L}\right\}$ which is the image of precisely three points of $X \backslash Q^{+}(3,3)$, each of the two remaining points of $L \backslash\left\{x_{L}\right\}$ is the image of precisely two points of $X \backslash Q^{+}(3,3)$. In this case, the point $x_{L}$ itself is not the image of any point of $X \backslash Q^{+}(3,3)$.

In case (a), we see that $x_{L}$ is the unique point of $U_{2}$ on $L$. In case (b), we see that $x_{L}^{\prime}$ is the unique point of $U_{2}$ on $L$. Since $L \cap U_{2}$ is always a singleton, we conclude that $U_{2}$ must be an ovoid of $Q^{+}(3,3)$.

Now, if $\mathcal{C}$ is the collection of the seven ovoids $O_{y}$, where $y \in X \backslash Q^{+}(3,3)$, then the following properties hold:

(P1) No point of $U_{1} \backslash U_{2}$ is contained in an ovoid of $\mathcal{C}$.

(P2) Every point of $U_{1} \cap U_{2}$ is contained in precisely one ovoid of $\mathcal{C}$.

(P3) Every point of $Q^{+}(3,3) \backslash\left(U_{1} \cup U_{2}\right)$ is contained in precisely two ovoids of $\mathcal{C}$.

(P4) Every point of $U_{2} \backslash U_{1}$ is contained in precisely three ovoids of $\mathcal{C}$.

(P5) No two ovoids of $\mathcal{C}$ intersect in a singleton $\{x\}$, where $x \in Q^{+}(3,3) \backslash\left(U_{1} \cup U_{2}\right)$.

(P6) No three ovoids of $\mathcal{C}$ can mutually intersect in the same singleton $\{x\}$, where $x \in$ $U_{2} \backslash U_{1}$

Proposition 4.8. Suppose that $U_{1}$ and $U_{2}$ are two (not necessarily distinct) ovoids of $Q^{+}(3,3)$. Let $Y$ be a set of seven points of $\mathrm{PG}(3,3) \backslash Q^{+}(3,3)$ and put $\mathcal{C}:=\left\{O_{y} \mid y \in Y\right\}$. If $\mathcal{C}$ satisfies the properties (P1) - (P6) above, then $U_{1} \cup Y$ is a $\mathcal{T}$-blocking set of size 11.

Proof. We have $\left|U_{1} \cup Y\right|=11$. Since $U_{1}$ is an ovoid of $Q^{+}(3,3)$, every $\mathcal{T}_{0}$-line meets $U_{1}$ at a unique point. Every $\mathcal{T}_{1}$-line through a point of $U_{1}$ obviously meets $U_{1}$. By (P4) and (P6), every $\mathcal{T}_{1}$-line through a point of $U_{2} \backslash U_{1}$ contains a point of $Y$. By (P3) and (P5), every $\mathcal{T}_{1}$-line through a point of $Q^{+}(3,3) \backslash\left(U_{1} \cup U_{2}\right)$ contains a point of $Y$.

We now use the above result to classify the $\mathcal{T}$-blocking sets of size 11 in $\operatorname{PG}(3,3)$. We assume that $U_{1}$ and $U_{2}$ are two ovoids of $Q^{+}(3,3)$ and that $\mathcal{C}$ is a collection of seven ovoids of $Q^{+}(3,3)$ satisfying the properties (P1) - (P6) above. If $Y$ is the set of seven points of $\mathrm{PG}(3,3) \backslash Q^{+}(3,3)$ for which the collection $\left\{O_{y} \mid y \in Y\right\}$ coincides with $\mathcal{C}$, then $X=U_{1} \cup Y$ is a $\mathcal{T}$-blocking set of size 11 by Proposition 4.8. Without loss of generality, we may suppose that $U_{1}=O^{*}=\left\{x_{11}, x_{22}, x_{33}, x_{44}\right\}$. Then the nine ovoids disjoint from $U_{1}=\left\{x_{11}, x_{22}, x_{33}, x_{44}\right\}$ are $O_{1}, O_{2}, \ldots, O_{9}$ as defined in the beginning of this section.

The ovoid $U_{2}$ can have five positions with respect to $U_{1}$ (up to isomorphism): 


$$
\begin{aligned}
& \text { I: } U_{2}=\left\{x_{11}, x_{22}, x_{33}, x_{44}\right\}=U_{1}, \\
& \text { II: } U_{2}=\left\{x_{11}, x_{22}, x_{34}, x_{43}\right\}, \\
& \text { III: } U_{2}=\left\{x_{11}, x_{23}, x_{34}, x_{42}\right\}, \\
& \text { IV: } U_{2}=\left\{x_{12}, x_{21}, x_{34}, x_{43}\right\}, \\
& \text { V: } U_{2}=\left\{x_{12}, x_{23}, x_{34}, x_{41}\right\} .
\end{aligned}
$$

\section{Treatment of Case I}

In this case, (P2) implies that the points of $U_{1} \cap U_{2}=U_{1}=U_{2}$ are partitioned by certain ovoids of $\mathcal{C}$. The partition has shape $4,2+2,2+1+1$ or $1+1+1+1$, leading to four subcases.

(Ia) Suppose the mentioned partition has shape 4. Then $U_{1}=U_{2} \in \mathcal{C}$. Again (P2) implies that every ovoid of $\mathcal{C} \backslash\left\{U_{1}\right\}$ is disjoint from $U_{1}=U_{2}$. By $(\mathrm{P} 3), \mathcal{C} \backslash\left\{U_{1}\right\}$ is a collection of six ovoids as in Lemma 4.1. A contradiction is then readily obtained from Lemma 4.2 and property (P5).

(Ib) Suppose the mentioned partition has shape $2+2$. Without loss of generality, we may suppose that $\left\{x_{11}, x_{22}, x_{34}, x_{43}\right\}$ and $\left\{x_{33}, x_{44}, x_{12}, x_{21}\right\}$ belong to $\mathcal{C}$. By (P2), each of the remaining five ovoids of $\mathcal{C}$ is disjoint from $U_{1}=U_{2}$. So we need to find five additional ovoids from the collection $\left\{O_{1}, O_{2}, \ldots, O_{9}\right\}$. By (P3) and (P5), the second ovoid of $\mathcal{C}$ through $x_{12}$ must contain $x_{21}$ and therefore be equal to $O_{1}=\left\{x_{12}, x_{21}, x_{34}, x_{43}\right\}$. As $x_{12}$, $x_{21}, x_{34}$ and $x_{43}$ have already been covered twice, the remaining four ovoids should be contained in $\left\{x_{13}, x_{14}, x_{23}, x_{24}, x_{31}, x_{32}, x_{41}, x_{42}\right\}$ and hence equal to $O_{2}, O_{3}, O_{6}$ and $O_{9}$. One readily verifies that the collection consisting of the seven ovoids $\left\{x_{11}, x_{22}, x_{34}, x_{43}\right\}$, $\left\{x_{33}, x_{44}, x_{12}, x_{21}\right\}, O_{1}, O_{2}, O_{3}, O_{6}$ and $O_{9}$ satisfies the properties $(\mathrm{P} 1)-(\mathrm{P} 6)$.

(Ic) Suppose the mentioned partition has shape $2+1+1$. Without loss of generality, we may suppose that $\left\{x_{11}, x_{22}, x_{34}, x_{43}\right\}$ is present in $\mathcal{C}$. Then the ovoid $\left\{x_{12}, x_{21}, x_{33}, x_{44}\right\}$ is not in $\mathcal{C}$. By (P3) and (P5), the second ovoid of $\mathcal{C}$ through $x_{34}$ must contain $x_{43}$ and hence coincides with $O_{1}=\left\{x_{12}, x_{21}, x_{34}, x_{43}\right\}$. Note that each of $x_{34}, x_{43}$ has now been covered twice, while each of $x_{12}$ and $x_{21}$ only once. Therefore the second ovoid of $\mathcal{C}$ through $x_{12}$, which cannot intersect $\left\{x_{12}, x_{21}, x_{34}, x_{43}\right\}$ in a singleton, must also contain $x_{21}$. But that is impossible as the two ovoids through $\left\{x_{12}, x_{21}\right\}$, namely $O_{1}$ and $\left\{x_{12}, x_{21}, x_{33}, x_{44}\right\}$ are already forbidden.

(Id) Suppose the mentioned partition has shape $1+1+1+1$. Without loss of generality, we may suppose that $\left\{x_{11}, x_{23}, x_{34}, x_{42}\right\}$ belongs to $\mathcal{C}$. Each $y \in\left\{x_{23}, x_{34}, x_{42}\right\}$ is contained in a second ovoid of $\mathcal{C}$ which meets $\left\{x_{11}, x_{23}, x_{34}, x_{42}\right\}$ in a second point $y^{\prime} \in\left\{x_{23}, x_{34}, x_{42}\right\}$. But then the pairs $\left\{y, y^{\prime}\right\}$ would partition $\left\{x_{23}, x_{34}, x_{42}\right\}$, an obvious contradiction. 


\section{Treatment of Case II}

We have $U_{2}=\left\{x_{11}, x_{22}, x_{34}, x_{43}\right\}$. If $U_{2} \in \mathcal{C}$, then by (P1) - (P4), $\mathcal{C} \backslash\left\{U_{2}\right\}$ is a collection of six ovoids as in Lemma 4.1. A contradiction is then readily obtained from Lemma 4.2 and property (P5). So, $U_{2} \notin \mathcal{C}$. By (P1) and (P2), it follows that the unique ovoid of $\mathcal{C}$ containing $x_{11}$ is either $\left\{x_{11}, x_{23}, x_{34}, x_{42}\right\}$ or $\left\{x_{11}, x_{24}, x_{32}, x_{43}\right\}$. In view of the symmetry $3 \leftrightarrow 4$, we may without loss of generality suppose that $\left\{x_{11}, x_{23}, x_{34}, x_{42}\right\}$ is the unique ovoid of $\mathcal{C}$ containing $x_{11}$. There are still six ovoids to choose for $\mathcal{C}$, one of them contains $x_{22}$ and the other five are contained in the collection $\left\{O_{1}, O_{2}, \ldots, O_{9}\right\}$. None of these six ovoids can intersect $\left\{x_{11}, x_{23}, x_{34}, x_{42}\right\}$ in the singleton $\left\{x_{23}\right\}$ or the singleton $\left\{x_{42}\right\}$, implying that $\mathrm{O}_{2}$ and $O_{3}$ do not belong to $\mathcal{C}$. So, we need to take five ovoids among the seven ovoids $O_{1}, O_{4}, O_{5}, O_{6}, O_{7}, O_{8}, O_{9}$. Since $O_{4} \cap O_{5}=\left\{x_{12}\right\}, O_{5} \cap O_{6}=\left\{x_{41}\right\}$, $O_{4} \cap O_{6}=\left\{x_{24}\right\}$ and $O_{7} \cap O_{9}=\left\{x_{42}\right\}$, (P5) implies that none of the pairs $\left\{O_{4}, O_{5}\right\}$, $\left\{O_{5}, O_{6}\right\},\left\{O_{4}, O_{6}\right\},\left\{O_{7}, O_{9}\right\}$ can be contained in $\mathcal{C}$. So, two among $O_{4}, O_{5}, O_{6}$ cannot be in $\mathcal{C}$, as well as one among $O_{7}, O_{9}$. So, it is impossible to find the five required ovoids from the collection $\left\{O_{1}, O_{4}, O_{5}, \cdots, O_{9}\right\}$.

\section{Treatment of Case III}

We have $U_{2}=\left\{x_{11}, x_{23}, x_{34}, x_{42}\right\}$. If $U_{2} \in \mathcal{C}$, then by $(\mathrm{P} 1)-(\mathrm{P} 4), \mathcal{C} \backslash\left\{U_{2}\right\}$ is a collection of six ovoids as in Lemma 4.1. A contradiction is then readily obtained from Lemma 4.2 and property (P5). So, $U_{2} \notin \mathcal{C}$. Then, using (P1) and (P2), the unique ovoid of $\mathcal{C}$ containing $x_{11}$ must be $\left\{x_{11}, x_{24}, x_{32}, x_{43}\right\}$. Each point $y \in\left\{x_{24}, x_{32}, x_{43}\right\}$ is contained in a second ovoid of the collection $\mathcal{C}$ which meets $\left\{x_{11}, x_{24}, x_{32}, x_{43}\right\}$ in a second point $y^{\prime} \in\left\{x_{24}, x_{32}, x_{43}\right\}$. Then the pairs $\left\{y, y^{\prime}\right\}$ would partition $\left\{x_{24}, x_{32}, x_{43}\right\}$, an obvious contradiction.

\section{Treatment of Case IV}

We have $U_{2}=\left\{x_{12}, x_{21}, x_{34}, x_{43}\right\}$. By (P1), all ovoids of $\mathcal{C}$ are disjoint from $U_{1}$. So we have to choose seven ovoids for $\mathcal{C}$ among the nine ovoids $O_{1}, O_{2}, \ldots, O_{9}$. By (P4), there are three ovoids of $\mathcal{C}$ containing $x_{12}$. So the ovoids $O_{1}, O_{4}$ and $O_{5}$ belong to $\mathcal{C}$. As $O_{4} \cap O_{6}=\left\{x_{24}\right\}$ and $O_{4} \cap O_{9}=\left\{x_{31}\right\}$, the ovoids $O_{6}$ and $O_{9}$ are not in $\mathcal{C}$ by (P5). Hence, $\mathcal{C}=\left\{O_{1}, O_{2}, O_{3}, O_{4}, O_{5}, O_{7}, O_{8}\right\}$. One readily verifies that this collection of ovoids satisfies the properties $(\mathrm{P} 1)-(\mathrm{P} 6)$.

\section{Treatment of Case V}

Here $U_{2}=\left\{x_{12}, x_{23}, x_{34}, x_{41}\right\}$. By $(\mathrm{P} 1)$, all ovoids of $\mathcal{C}$ are disjoint from $U_{1}$. So we have to choose seven ovoids for $\mathcal{C}$ among the nine ovoids $O_{1}, O_{2}, \ldots, O_{9}$. Since $O_{4} \cap O_{6}=\left\{x_{24}\right\}$, $O_{4} \cap O_{8}=\left\{x_{43}\right\}$ and $O_{4} \cap O_{9}=\left\{x_{31}\right\}, O_{4}$ cannot occur in $\mathcal{C}$ by (P5). Since $O_{6} \cap O_{7}=\left\{x_{13}\right\}$ and $O_{6} \cap O_{8}=\left\{x_{32}\right\}$, we then know that also $O_{6}$ cannot occur in $\mathcal{C}$. So, we should have that $\mathcal{C}=\left\{O_{1}, O_{2}, O_{3}, O_{5}, O_{7}, O_{8}, O_{9}\right\}$. But that is impossible again by (P5) as $O_{7} \cap O_{8}=\left\{x_{21}\right\}$. 
Let $X_{1}=U_{1} \cup Y_{1}=O^{*} \cup Y_{1}$, where $Y_{1}$ is the set of seven points of $\mathrm{PG}(3,3) \backslash$ $Q^{+}(3,3)$ for which the collection $\left\{O_{y} \mid y \in Y_{1}\right\}$ consists of the ovoids $\left\{x_{11}, x_{22}, x_{34}, x_{43}\right\}$, $\left\{x_{33}, x_{44}, x_{12}, x_{21}\right\}, O_{1}, O_{2}, O_{3}, O_{6}$ and $O_{9}$ of $Q^{+}(3,3)$. Similarly, let $X_{2}=U_{1} \cup Y_{2}=$ $O^{*} \cup Y_{2}$, where $Y_{2}$ is the set of seven points of $\mathrm{PG}(3,3) \backslash Q^{+}(3,3)$ for which the collection $\left\{O_{y} \mid y \in Y_{2}\right\}$ coincides with $\left\{O_{1}, O_{2}, O_{3}, O_{4}, O_{5}, O_{7}, O_{8}\right\}$. Note that $X_{1}$ is associated with the seven ovoids corresponding to subcase (Ib) in the treatment of Case I and $X_{2}$ is associated with the seven ovoids in the treatment of Case IV.

By the above discussion, we thus know:

Proposition 4.9. Up to isomorphism, $X_{1}$ and $X_{2}$ are the two $\mathcal{T}$-blocking sets of size 11 in $\mathrm{PG}(3,3)$.

Our intention is now to identify the two blocking sets $X_{1}$ and $X_{2}$ with that of $B_{1}$ and $B_{2}$ constructed, respectively, in Sections 3.1 and 3.2. We shall rely on the following lemma.

Lemma 4.10. Every ovoid $O$ of $Q^{+}(3,3)$ is contained in precisely four partitions of $Q^{+}(3,3)$ into ovoids. Three of these are induced by external lines.

Proof. Without loss of generality, we may suppose that $O=O^{*}$. The partitions then have the form $\left\{O^{*}, O_{i}, O_{j}, O_{k}\right\}$, where $i, j, k \in\{1,2, \ldots, 9\}$ with $i<j<k$. It is straightforward to verify that these partitions are $\left\{O^{*}, O_{1}, O_{2}, O_{3}\right\},\left\{O^{*}, O_{1}, O_{6}, O_{9}\right\},\left\{O^{*}, O_{2}, O_{5}, O_{8}\right\}$ and $\left\{O^{*}, O_{3}, O_{4}, O_{7}\right\}$. Now, let $x$ denote the unique point of $\mathrm{PG}(3,3) \backslash Q^{+}(3,3)$ for which $O_{x}=O=O^{*}$. There are three external lines through $x$. If $\left\{x, u_{1}, u_{2}, u_{3}\right\},\left\{x, u_{4}, u_{5}, u_{6}\right\}$ and $\left\{x, u_{7}, u_{8}, u_{9}\right\}$ are these external lines, then the nine ovoids $\left\{O_{u_{1}}, O_{u_{2}}, \ldots, O_{u_{9}}\right\}$ are mutually distinct. So, $\left\{O^{*}, O_{1}, O_{6}, O_{9}\right\},\left\{O^{*}, O_{2}, O_{5}, O_{8}\right\}$ and $\left\{O^{*}, O_{3}, O_{4}, O_{7}\right\}$ must be the partitions among the four that are induced by external lines.

Proposition 4.11. There exist two mutually disjoint external lines $K, L$ and a point $x \in K$ such that $X_{1}=O_{x} \cup(K \backslash\{x\}) \cup L$.

Proof. Let $K$ denote the external line determined by the ovoids $O^{*}, O_{1}, O_{6}, O_{9}$, and denote by $x$ the unique point of $K$ for which $O_{x}=O^{*}$. Among the four partitions of $Q^{+}(3,3)$ into ovoids containing $O_{2},\left\{O^{*}, O_{1}, O_{2}, O_{3}\right\}$ is not induced by any external line (see the proof of Lemma 4.10). So, again by Lemma 4.10, the partition of $Q^{+}(3,3)$ by the ovoids $\left\{x_{11}, x_{22}, x_{34}, x_{43}\right\},\left\{x_{33}, x_{44}, x_{12}, x_{21}\right\}, O_{2}, O_{3}$ is induced by some external line, say $L$. Then we have $K \cap L=\emptyset$ and $X_{1}=O_{x} \cup(K \backslash\{x\}) \cup L$.

By Proposition 3.3, we know that the two blocking sets $B_{1}$ and $B_{2}$ constructed in Sections 3.1 and 3.2 are nonisomorphic. In fact, by the proof of that proposition, we know that $B_{2}$ does not contain any external line, while $B_{1}$ does. Comparing this with Propositions 4.9 and 4.11 , we then conclude that the blocking set $X_{1}$ is isomorphic to $B_{1}$ and that the blocking set $X_{2}$ is isomorphic to $B_{2}$. 


\section{References}

[1] P. Biondi and P. M. Lo Re, On blocking sets of external lines to a hyperbolic quadric in $\mathrm{PG}(3, q), q$ even, J. Geom. 92 (2009), 23-27.

[2] P. Biondi, P. M. Lo Re and L. Storme, On minimum size blocking sets of external lines to a quadric in PG(3,q), Beiträge Algebra Geom. 48 (2007), 209-215.

[3] A. Bishnoi and B. De Bruyn, On generalized hexagons of order $(3, t)$ and $(4, t)$ containing a subhexagon, European J. Combin. 62 (2017), 115-123.

[4] R. C. Bose and R. C. Burton, A characterization of flat spaces in a finite geometry and the uniqueness of the Hamming and the MacDonald codes, J. Combinatorial Theory 1 (1966), 96-104.

[5] B. De Bruyn, B. K. Sahoo and B. Sahu, Computer computations for "Blocking sets of tangent lines to a hyperbolic quadric in $\mathrm{PG}(3,3)$ ", available online at https: //cage.ugent.be/geometry/preprints.php

[6] J. W. P. Hirschfeld, Finite Projective Spaces of Three Dimensions, Oxford University Press, Oxford, 1985.

[7] G. E. Moorhouse, Incidence Geometry, 2007, available online at http://www . uwyo. edu/moorhouse/handouts/incidence_geometry.pdf

[8] S. E. Payne and J. A. Thas, Finite Generalized Quadrangles, EMS series of lectures in Mathematics, European Mathematical Society, Zurich, 2009.

[9] Sage Mathematics Software (Version 6.3), The Sage Developers, 2014, http://www. sagemath.org.

[10] B. K. Sahoo and B. Sahu, Blocking sets of tangent and external lines to a hyperbolic quadric in $\mathrm{PG}(3, q), q$ even, Proc. Indian Acad. Sci. Math. Sci., to appear.

[11] B. K. Sahoo and B. Sahu, Blocking sets of certain line sets to a hyperbolic quadric in $\mathrm{PG}(3, q)$, submitted.

[12] B. K. Sahoo and N. S. N. Sastry, Binary codes of the symplectic generalized quadrangle of even order, Des. Codes Cryptogr. 79 (2016), 163-170.

[13] The GAP Group, GAP - Groups, Algorithms, and Programming, Version 4.7.5, 2014. (http://www.gap-system.org)

\section{Addresses:}

Bart De Bruyn

Department of Mathematics, Ghent University 
Krijgslaan 281 (S22), B-9000 Gent, Belgium

Email: Bart.DeBruyn@Ugent.be

\section{Binod Kumar Sahoo and Bikramaditya Sahu}

School of Mathematical Sciences

National Institute of Science Education and Research, Bhubaneswar (HBNI)

P.O. - Jatni, District- Khurda, Odisha - 752050, India

Emails: bksahoo@niser.ac.in, bikram.sahu@niser.ac.in 